\title{
ARTICLE
}

Acute myeloid leukemia

\section{MYC-containing amplicons in acute myeloid leukemia: genomic structures, evolution, and transcriptional consequences}

\author{
Alberto L'Abbate ${ }^{1} \cdot$ Doron Tolomeo $^{1} \cdot$ Ingrid Cifola $^{2} \cdot$ Marco Severgnini $^{2}$ - Antonella Turchiano ${ }^{1}$. \\ Bartolomeo Augello ${ }^{3}$ - Gabriella Squeo ${ }^{3}$ - Pietro D'Addabbo $\mathbb{D}^{1} \cdot$ Debora Traversa $^{1} \cdot$ Giulia Daniele ${ }^{1}$. \\ Angelo Lonoce ${ }^{1} \cdot$ Mariella Pafundi $^{1} \cdot$ Massimo Carella $^{3} \cdot$ Orazio Palumbo $\mathbb{D}^{3} \cdot$ Anna Dolnik $^{4}$. \\ Dominique Muehlematter ${ }^{5}$. Jacqueline Schoumans ${ }^{5} \cdot$ Nadine Van Roy $^{6} \cdot$ Gianluca De Bellis $^{2} \cdot$ Giovanni Martinelli $^{7}$. \\ Giuseppe Merla ${ }^{3} \cdot$ Lars Bullinger $^{4} \cdot$ Claudia Haferlach $^{8} \cdot$ Clelia Tiziana Storlazzi $^{1}$
}

Received: 4 August 2017 / Revised: 27 October 2017 / Accepted: 13 November 2017 / Published online: 22 February 2018

(c) The Author(s) 2018. This article is published with open access

\begin{abstract}
Double minutes (dmin), homogeneously staining regions, and ring chromosomes are vehicles of gene amplification in cancer. The underlying mechanism leading to their formation as well as their structure and function in acute myeloid leukemia (AML) remain mysterious. We combined a range of high-resolution genomic methods to investigate the architecture and expression pattern of amplicons involving chromosome band 8q24 in 23 cases of AML (AML-amp). This revealed that different $M Y C$-dmin architectures can coexist within the same leukemic cell population, indicating a step-wise evolution rather than a single event origin, such as through chromothripsis. This was supported also by the analysis of the chromothripsis criteria, that poorly matched the model in our samples. Furthermore, we found that dmin could evolve toward ring chromosomes stabilized by neocentromeres. Surprisingly, amplified genes (mainly PVT1) frequently participated in fusion transcripts lacking a corresponding DNA template. We also detected a significant overexpression of the circular RNA of PVT1 (circPVT1) in AML-amp cases versus AML with a normal karyotype. Our results show that 8q24 amplicons in AML are surprisingly plastic DNA structures with an unexpected association to novel fusion transcripts and circular RNAs.
\end{abstract}

Electronic supplementary material The online version of this article (https://doi.org/10.1038/s41375-018-0033-0) contains supplementary material, which is available to authorized users.

Clelia Tiziana Storlazzi

cleliatiziana.storlazzi@uniba.it

1 Department of Biology, University of Bari “Aldo Moro", Bari, Italy

2 Institute for Biomedical Technologies (ITB), CNR, Segrate, Italy

3 Division of Medical Genetics, IRCCS 'Casa Sollievo della Sofferenza', San Giovanni Rotondo, Italy

4 Department of Internal Medicine III, University Hospital of Ulm, Ulm, Germany

5 Centre Hospitalier Universitaire Vaudois (CHUV), Lausanne University Hospital, Lausanne, Switzerland

6 Center for Medical Genetics, Ghent University, Ghent, Belgium

7 Istituto Scientifico Romagnolo per lo Studio e la Cura dei Tumori (IRST) IRCCS, Meldola, Italy

8 Munich Leukemia Laboratory (MLL), Munich, Germany

\section{Introduction}

Genomic amplifications in the form of double minutes (dmin), homogeneously staining regions (hsr), and ring chromosomes are frequent in cancer [1], although rare in leukemia $(<1 \%$ of cytogenetically abnormal hematological malignancies) [2], and associated with an elderly age and poor prognosis [3, 4]. Despite this clinical impact, the underlying mechanisms of amplicon formation in acute myeloid leukemia (AML) are unclear, and so are their internal structures. Chromothripsis was described as a potential mechanism involved in their genesis [5, 6]. However, the evidence we previously obtained in seven tumor cell lines harboring 8q24 amplifications clearly excluded this model in our samples [7], supporting alternative mechanisms such as the episome model [8-10]. Another crucial aspect of amplicons is their impact on the transcriptome. Amplified cancer-associated genes are often upregulated as a direct consequence of their copy number (CN) gain [11]. Nevertheless, MYC, as well as other 8q24 co-amplified genes and long noncoding RNAs (lncRNAs) (e.g., TRIB1, FAM84B, POU5F1B, PVT1, and TMEM75), 
represents a clear exception to this rule in myeloid malignancies $[9,12,13]$. These findings suggest that none of them could be considered as the real target for this type of amplification [14].

We investigated, at both genomic and transcriptomic levels, a large cohort of AML cases harboring MYC amplifications in the form of dmin, hsr, or ring chromosomes (AML-amp), in order to unravel the molecular mechanisms as well as potential target genes behind their genesis. To this end, we combined fluorescence in situ hybridization (FISH), immuno-FISH, PCR, singlenucleotide polymorphism (SNP) array, whole-genome sequencing (WGS), and RNA sequencing (RNA-seq). Overall, our study provides a full overview of the 8q24 amplicon organization in $\mathrm{dmin} / \mathrm{hsr}$, as well as on ring chromosomes, and sheds light on the extraordinarily high transcriptome plasticity associated with AML-amp.

\section{Materials and methods}

\section{Sample collection}

This study included a total of 24 AML-amp cases (Supplementary Table S1), two remission samples from cases that were AML-amp at diagnosis, 29 AML cases with a normal karyotype (AML-NK), 10 AML cases with chromosomal abnormalities not involving chromosome 8, 23 AML cell lines with or without $8 \mathrm{q} 24$ gain, and one normal peripheral blood (PB) and one bone marrow (BM) sample as controls (Supplementary Table S1 and Supplementary Methods).

\section{Neocentromere detection on ring chromosomes}

AML-amp cases MLL_11929 and MLL_11933, as well as two additional ones (\#18 and \#33) included in a previously described cohort [9], with ring chromosomes were investigated for the presence of neocentromere using FISH cohybridization experiments with an anti-CENP-C polyclonal antibody (Cat. no. ABIN3072962 Eurogentec polyclonal antibody service, Aachen, Germany) for functional centromeres [15], a pan-centromeric probe detecting all types of alphoid subset sequences [16], and a BAC probe (RP11440 N18) specific for $M Y C$.

\section{SNP array analysis}

SNP array analysis was performed on 24 AML-amp cases using the Affymetrix CytoScan HD platform (Affymetrix, Santa Clara, CA, USA) following the manufacturer's protocol.

\section{WGS and identification of structural variants}

WGS was performed on 23 AML-amp cases and two remission samples (Supplementary Table S1) using the $\mathrm{X}$ Ten platform (Illumina, San Diego, CA, USA), in a pairedend $2 \times 150$-cycle run, with a mean coverage of $30 \times$ per sample. Candidate structural variants (SVs) were identified using the DELLY software (v.0.7.1) [17] (details are provided in Supplementary Methods).

\section{Chromothripsis analysis}

To infer chromothripsis, WGS and SNP array profiles of each case were evaluated according to published criteria [18] (Supplementary Methods). We focused on the region 8q22.3-24.1 involved in the amplifications.

\section{RNA-seq analysis}

RNA-seq was performed on 20 AML-amp cases and two remission samples (Supplementary Table S1). RNA-seq libraries were prepared using the TruSeq RNA Sample Preparation Kit v2 (Illumina) and sequenced on the HiSeq2000 platform (Illumina) in paired-end $2 \times 101$-cycle runs (166 Mreads/sample). To detect fusion transcripts involving amplified genes, we used both ChimeraScan (CS) [19] and FusionMap (FM) [20] (Supplementary Methods). A custom bioinformatics pipeline was implemented to combine SVs with chimeric transcript data and evaluate the occurrence of post-transcriptional events in the genesis of chimeras.

\section{Differential gene expression analysis}

To ascertain genes specifically expressed only in AMLamp, six cases harboring 8q24 amplicons with no other chromosomal changes were compared with six AML-NK cases from The Cancer Genome Atlas (TCGA) (Supplementary Table S1). The DESeq2 Bioconductor package (v1.8.1) in R environment (v3.2.2) was used for Differential gene expression analysis (DEA) of global gene expression profiles in cases with/without $8 \mathrm{q} 24$ amplicons as well as for explorations of 8q24 genes in the AML-amp data set (Supplementary Methods). The ToppGene suite was used to perform gene enrichment analysis (https://toppgene.cchmc. org/).

\section{Mutation analysis of 8q24-amplified genes}

Genome and transcriptome data of 20 AML-amp cases (Supplementary Table S1) were analyzed in parallel for detection of non-synonymous single-nucleotide variants (SNVs) and insertions/deletions (indels). Variants were 
called using GATK HaplotypeCaller [21] and VarScan2 (VarScan 2.3.7) [22] on RNA-seq and WGS data, respectively, and then filtered according to specific criteria (Supplementary Methods). Pathway analysis was performed using the MSigDB [23], GSEA [24], and DAVID [25] tools to identify pathways associated with the mutated genes.

\section{Assessment of circular PVT1 (circPVT1) expression level}

The expression of both linear and $\operatorname{circPVTl}$ in eight AMLamp cases, 12 AML cell lines, nine AML-NK cases, and in one normal PB and one BM was analyzed by qPCR using properly designed primers [26]. The nine AML-NK samples were pooled and used as a calibrator; beta-2-microglobulin $(B 2 M)$ was used as a reference gene. Statistical significance was evaluated using the relative expression software tool REST [27].

\section{Results}

\section{$8 q 24 \mathrm{dmin} / \mathrm{hsr} /$ rings amplify a $2246 \mathrm{kbp}$ genomic segment encompassing 11 IncRNAs and four coding genes}

By combining SNP array, WGS and FISH analyses, we defined the 8q24 amplicon organization in 23 AML-amp cases. We identified a total of 373,585 raw SVs, of which 4630 were within the $8 \mathrm{q} 24$-amplified regions (Supplementary Table S2) and 84 of this subset (2\%) were considered as crucial for amplicon assembly, since they corresponded to $\mathrm{CN}$ switches. Their validation by PCR and Sanger sequencing revealed the occurrence of 30 microhomology sequences, 36 anonymous insertions, and 18 blunt-end joining events.

All AML-amp cases harbored amplified sequences derived only from chromosome 8 . The 8q24 amplifications were mainly present on dmin or ring chromosomes, and only rarely on hsr (Supplementary Table S3). The proximal and distal breakpoints of the amplicons clustered in two regions: the former at chr8:125,754,780-126,442,227 (687 $\mathrm{kbp})$ in $20 / 23$ (87\%) cases and the latter at chr8:130,690,866-130,950,708 (260 kbp) in 21/23 (91\%) cases. The minimal commonly amplified segment was 2,245,660 bp (Supplementary Figure S1). It comprises 15 RefSeq loci, including 11 lncRNAs (LINC00861, LOC101927657, PCAT1, PCAT2, PRNCR1, CASC19, CCAT1, CASC21, CASC8, CCAT2, and CASC11) and four coding genes (TRIB1, FAM84B, POU5F1B, and MYC). In addition, the lncRNAs $P V T 1$ and $C C D C 26$, just outside the commonly amplified region, were amplified in 22/24 (92\%) cases.
$8 q 24$ amplicon structures suggest different levels of molecular heterogeneity

All 23 cases investigated by WGS were classified according to the heterogeneity of $8 \mathrm{q} 24$ amplicons. In 21 out of 23 cases, we identified a most represented "ancestral" amplicon, which was repeated in a head-to-tail fashion.

Eight cases (35\%) displayed a single ancestral amplicon, ranging from $3216 \mathrm{kbp}$ to $5196 \mathrm{kbp}$, without remarkable switches in their $\mathrm{CN}$ profiles. These cases were considered to harbor non-heterogeneous amplicons (Fig. 1a, Supplementary Figure S2a-g). A duplication SV joined the distal and the proximal breakpoints of two adjacent amplified segments in all cases except MLL_11932 and MLL_11937 (Supplementary Figure S2a-g).

The remaining 15 of the 23 cases showed different levels of amplicon heterogeneity, which we dichotomized into lowgrade heterogeneity (LH) and high-grade heterogeneity (HH). In total, 12/23 (52\%) cases were classified as LH based on the co-existence of heterogeneous amplicons related to, and derived from, the ancestral amplified segment through the occurrence of internal SVs (Fig. 1b, Supplementary Figure S2h-r). Notably, all LH amplicons shared proximal and distal breakpoints in the same patient (Fig. 1b).

Three $(13 \%)$ of the 23 cases were considered $\mathrm{HH}$ because they comprised distinct cell subpopulations carrying amplicons with different internal structures arranged in a noncontiguous, noncollinear array (Fig. 2, Supplementary Figure S2s-t, Supplementary Methods).

\section{Complex structures at the deletions junctions on one chromosome 8 homolog}

Of the 23 cases analyzed by SNP array, WGS, and FISH, 11 $(48 \%)$ had heterozygous $8 \mathrm{q} 24$ deletions, corresponding to the amplified regions on the dmin, ring chromosomes, and hsr, on one of the chromosome 8 homologs. In four of the 11 cases, the deletion junctions were more complex than the ones in the other deleted cases, by displaying inversions with or without duplications, or reciprocal translocations at the junctions.

\section{Chromothripsis is not involved in 8q24 amplicon genesis}

The criteria defined to infer chromothripsis were evaluated across the 8q22.3-24.1 region in each AML-amp case (Table 1). According to the criterion "clustering of breakpoints", we searched for 5-10 breakpoints within $50 \mathrm{kbp}$ genomic segments. This criterion was fulfilled in $22 / 23$ (95.7\%) cases (Supplementary Figure S3a). Regarding the "regularity of oscillating copy number states", we did not observe more than $10 \mathrm{CN}$ switches in $16 / 23(69.6 \%)$ cases 


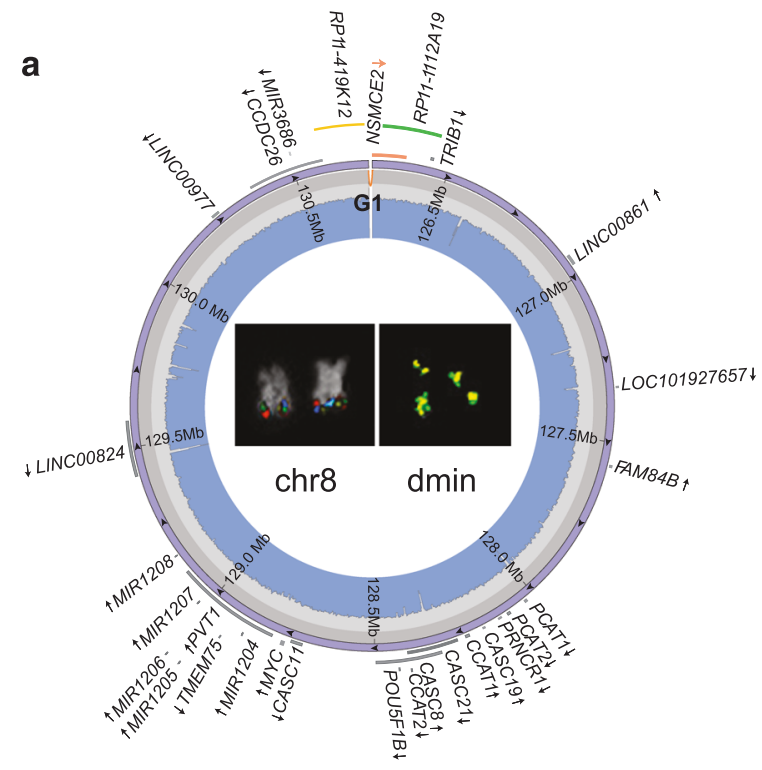

MLL_11940
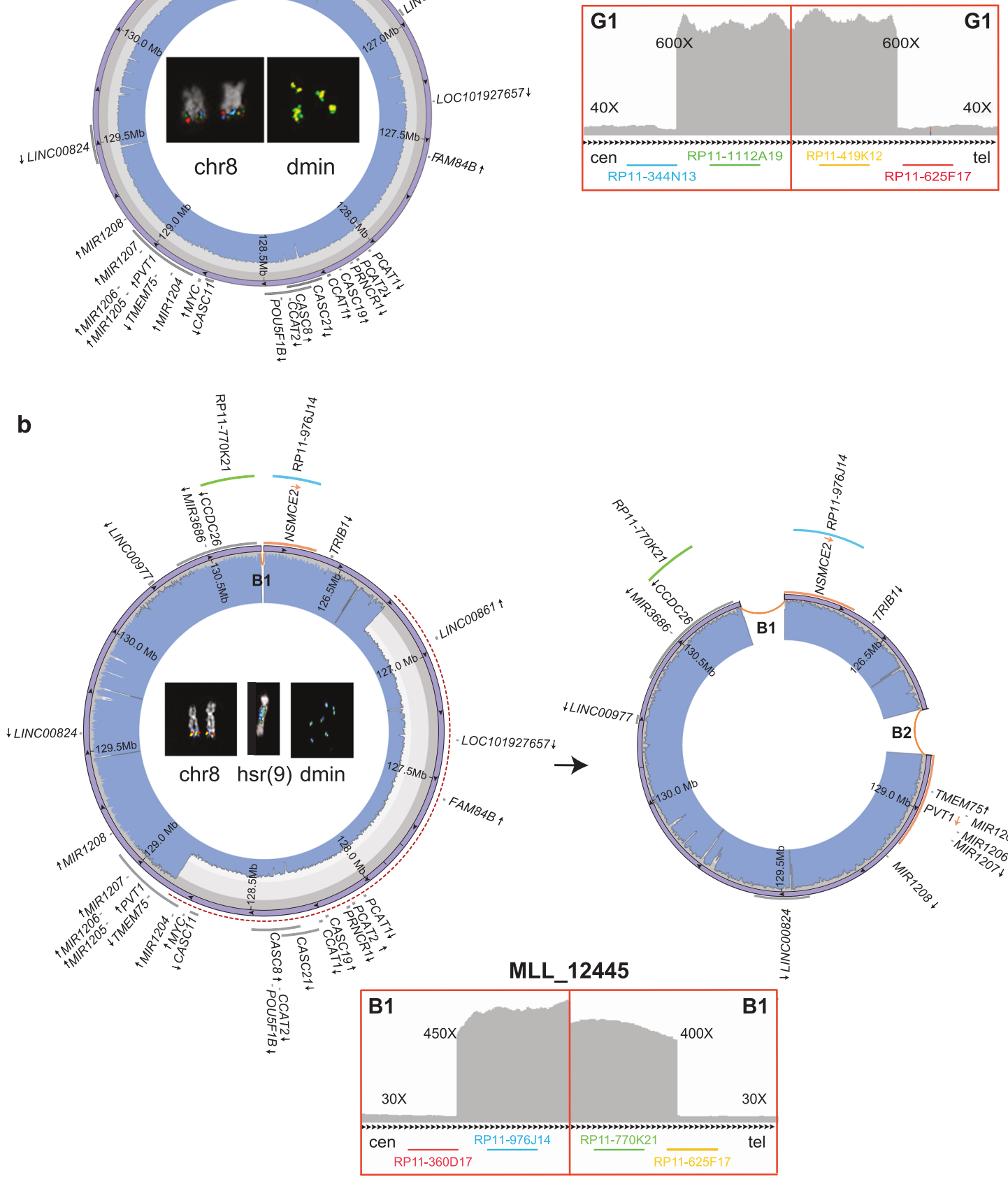

Fig. 1 Circular amplicons with no or low heterogeneity of structure. Images show the internal structure of amplified segments in cases MLL_11940 (as an example of a patient with no heterogeneous amplicons joined by a duplication) (a) and MLL_12445 (as an example of patient with two coexisting amplicons, one of which derived from the other by a deletion) (b). For each patient: IGV plot of WGS read depth at proximal and distal breakpoints of ancestral amplicons; Circos plots (outer violet circular panel) and WGS read depth (light blue inner panel) of recurrent amplicons, with internal arrows indicating amplicon orientation and genes in dark gray, or in orange if interrupted by a breakpoint (arrows represent the transcriptional orientation). Inside the Circos plots, FISH pseudocolor images showing co-localizing amplified probes, consistently colored as in the Circos plot. Probes mapping respectively upstream and downstream of the amplicons proximal and distal breakpoints are not amplified on dmin and display signal only on normal chromosome 8 . The reddashed semicircles in $\mathbf{b}$ represent sequence deletion on the ancestral amplicon to obtain the secondary one 
(Supplementary Figure S3b). In the remaining seven (30.4\%) cases, the $\mathrm{CN}$ values ranged between more than three $\mathrm{CN}$ states, thus exceeding the limit for chromothripsis.
Interestingly, we observed loss of heterozygosity in notdeleted regions, indicating the occurrence of multi-step rearrangement events (Supplementary Figure S3b).

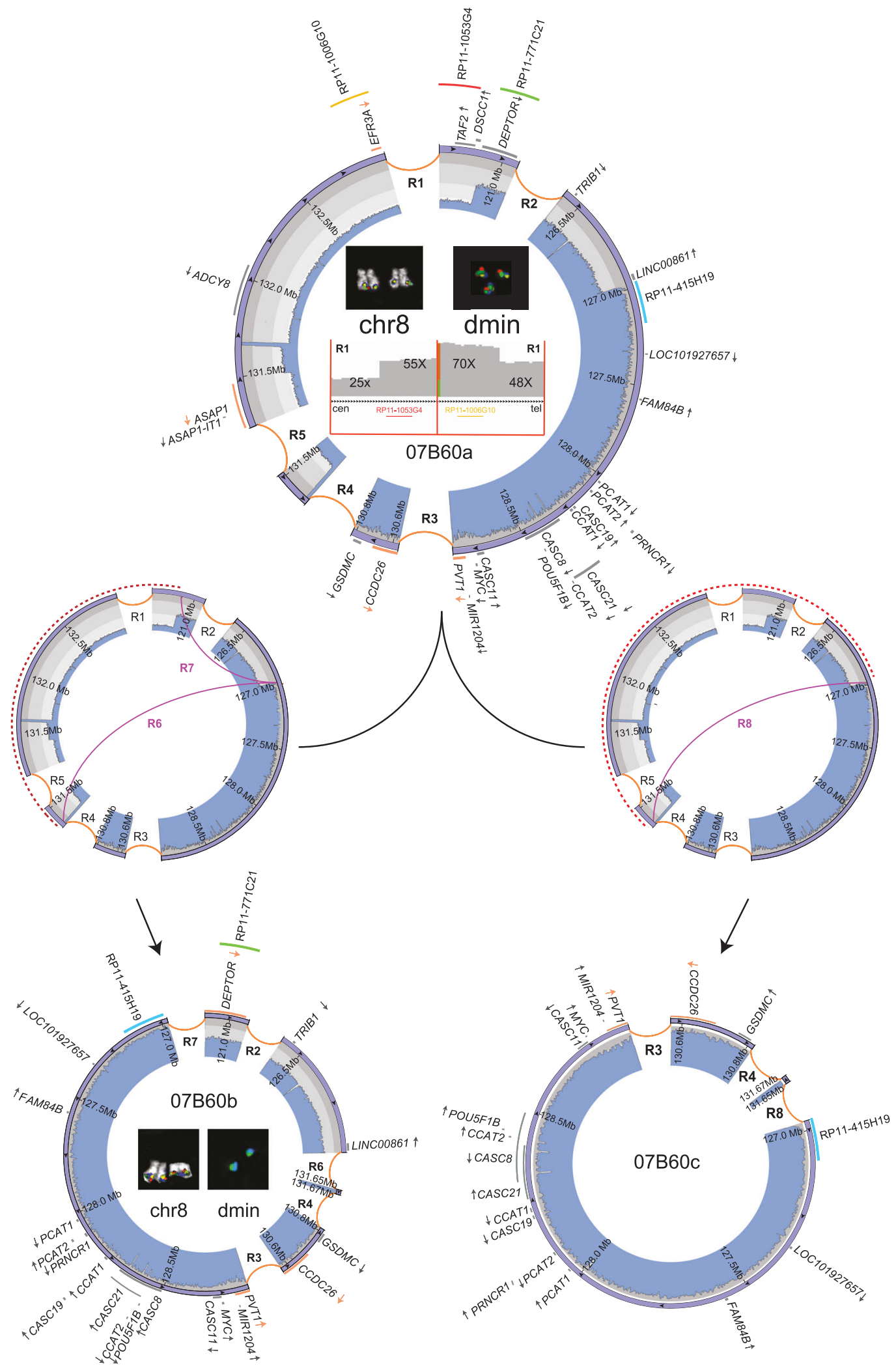


Concerning the "randomness of DNA segments order and fragment joins", the results were conflicting. On one hand, the order of the breakpoints was not conserved, indicating the presence of randomly joined chromosome fragments as in chromothripsis (Supplementary Figure S3c). On the other hand, there was not an equal distribution of rearrangement types, as occurs in the step-wise rearrangement model (Supplementary Figure S3d). Therefore, we considered this criterion as not validated for the assessment of chromothripsis in our samples. Finally, the criterion of "ability to walk the derivative chromosome" was not fulfilled because of the presence of nested amplified fragments (Supplementary Figure S3e); this does not fit the chromothripsis model in which no "reuse" of rearranged fragments is admitted. Taken together, the findings exclude the involvement of chromothripsis in the genesis of $8 \mathrm{q} 24$ amplicons.

\section{Neocentromeres stabilize tandemly amplified sequences within ring chromosomes}

FISH analyses of four AML-amp cases with amplicon-positive chromosomes revealed that the the primary constrictions on the ring chromosomes lacked alphoid satellite sequences but were positive for CENP-C (Fig. 3), strongly indicating the presence of functional ectopic centromeres, which are essential for stabilizing ring chromosomes during cell divisions.

\section{Amplified genes/IncRNAs are recurrently involved in fusion transcripts}

RNA-seq of AML-amp cases identified 12,468 and 58,032 raw chimeric transcripts by CS and FM, respectively, among which 38 and 429 involved 8q24-amplified genes (Supplementary Table S4A-B). Using RT-PCR and Sanger sequencing, we validated 35 of these 8q24 fusion transcripts (Table 2), 26 of which involved PVT1 as either a $5^{\prime}$ or $3^{\prime}$ partner (Supplementary Figure S4a). Notably, 15 chimeras with $P V T 1$ as a $5^{\prime}$ partner shared exactly the same breakpoint position (at chr8:128,806,980). Other genes/lncRNAs recurrently involved in chimeras were MYC, FAM49B, RP11-89K10, CCDC26, CASC11, and CASC8. Of these,

Fig. 2 Circular amplicons with high heterogeneity of structure. Images show the internal organization and inferred evolutionary path of amplicons in case 07B60, as an example of a patient with clonal amplicon heterogeneity. The ancestral amplicon (07B60a, occurring in the $26.4 \%$ (14/53) of cells), at the top of the figure, underwent two independent rearrangement types, indicated as R6/R7 (middle left) or R8 (middle right), originating the secondary amplicons 07B60b (bottom left), observed in $73.6 \%$ (39/53) of cells, and 07B60c (bottom right), without cell specificity at the FISH level. Circos plots (in violet) represent all reconstructed amplified structures, with WGS read depth (in light blue) and genes in dark gray, or in orange if interrupted by a breakpoint (arrows represent the transcriptional orientation). Red-
MYC and $C C D C 26$ each showed a recurrent breakpoint position (at chr8:128,750,494 and chr8:130,365,226, respectively) in three chimeras, where they are involved as $3^{\prime}$ partners. Remarkably, 15 out of the 35 (43\%) validated chimeras were generated by the fusion of two lncRNAs. Some fusion transcripts were common to two or more cases, were present as multiple transcript isoforms in the same case or in different cases, or together with their reciprocal fusion product (Table 2, Supplementary Table S4a).

In silico translation of the $5^{\prime} P V T 1$ chimeras predicted the dysregulation of the protein product of their $3^{\prime}$ partner transcripts due to promoter swapping or $\mathrm{N}$-terminus truncation (Table 2, Supplementary Figure S4b). Conversely, $3^{\prime}$ PVT1 fusions were predicted to display either a $3^{\prime}$ untranslated region substitution of their $5^{\prime}$ partner transcripts or a C-terminus truncation of the encoded protein. A truncation effect on the MTSS1 protein was also predicted for $5^{\prime} M T S S 1 / 3^{\prime} F A M 49 B$ fusion and its reciprocal $5^{\prime}$ FAM49B/3'MTSS1.

Interestingly, the $5^{\prime} P V T 1 / 3^{\prime} C C D C 26$ chimera was the only fusion we detected that was also in a panel of $23 \mathrm{AML}$ cell lines (Supplementary Table S4C-D). Similarly, neither the two remission samples of AML-amp cases nor the AML-NK cases harbored any of the 8q24 fusions detected in AML-amp cases.

\section{Fusion transcripts often do not correspond to genomic rearrangements}

By combining WGS and RNA-seq data, only $0.1 \%$ (12/ $12,468)$ and $0.07 \%(43 / 58,032)$ of the chimeric transcripts identified in our cohort by CS and FM, respectively, were detected at the genomic level. After zooming into the 8q24 chromosome segment, their percentages increased to $10.5 \%$ (4/38) and $7.2 \%$ (31/428) by CS and FM, respectively. These results suggest that the chimeric transcripts might be mostly generated by post-transcriptional events (Supplementary Table S4E). Moreover, our AML-amp cases showed a statistically significant higher number of $8 \mathrm{q} 24$ chimeras (mean 2.5 and 41.8 for CS and FM tools, respectively) as compared to AML-NK control cases from

dashed semicircles and purple curved lines respectively represent sequence deletions and newly originated SVs labeled by appropriated codes. Inside the 07B60a Circos plot, IGV plots of WGS read depth at amplicon borders (bottom) and FISH pseudocolor images show coamplification of delimiting amplicons proximal and distal breakpoints (top). The red-dashed semicircles represent sequence deletions on the ancestral amplicon to obtain both secondary ones. It is worth noting that dmin-harboring 07B60a amplicons showed the co-localization of all four indicated probes in the FISH experiments, contrary to those containing 07B60b, which displayed signals only for the green and blue probes, indicating a cell specificity of sequence amplifications 


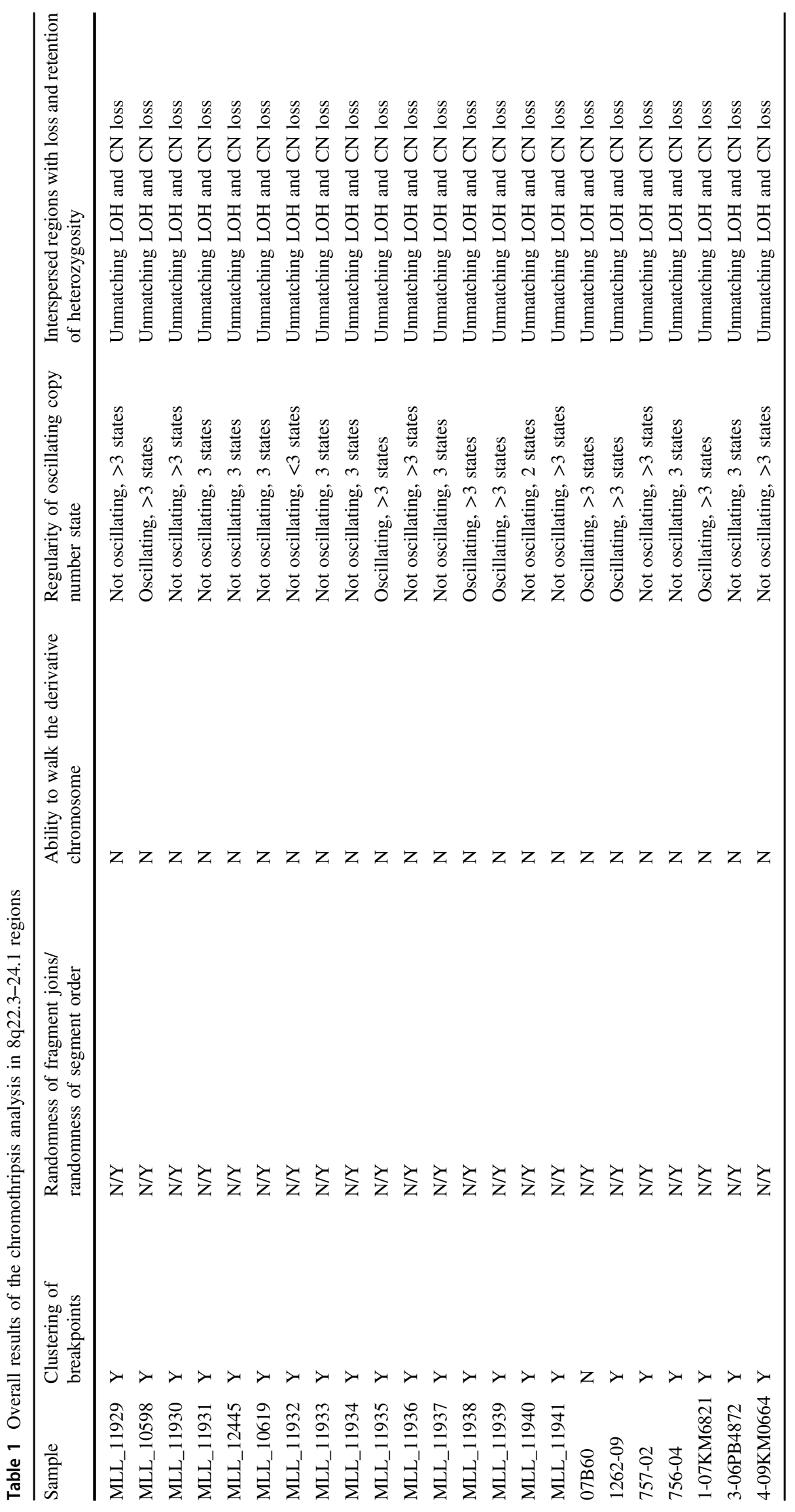




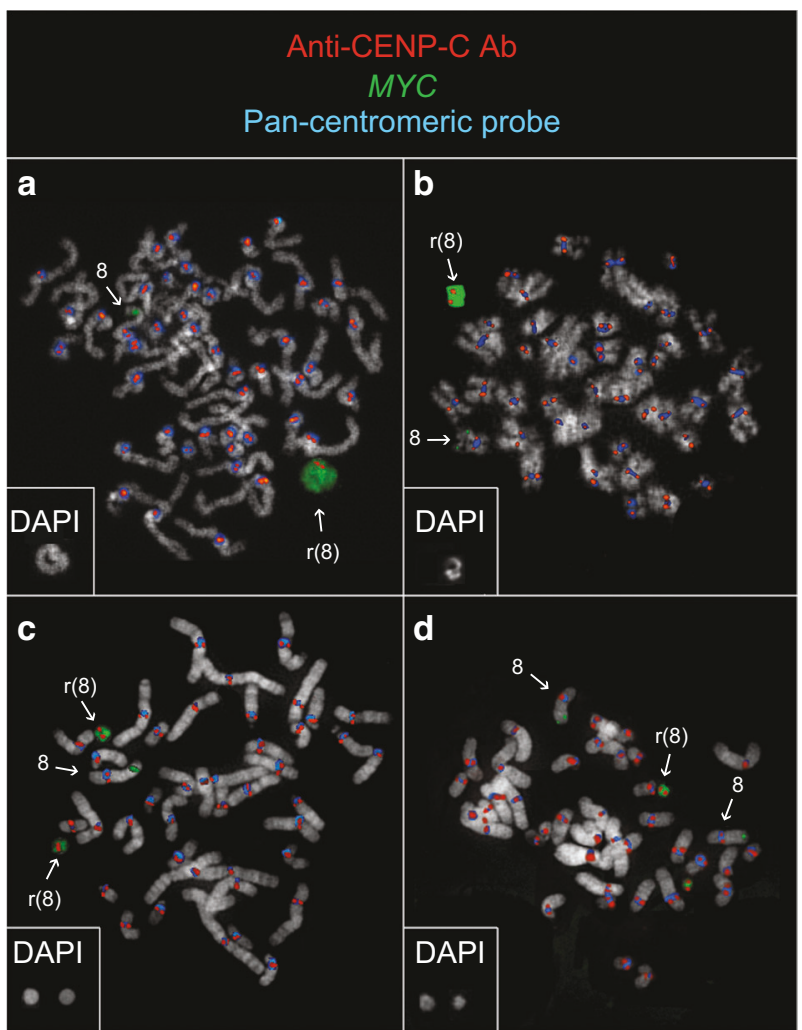

Fig. 3 Neocentromere at ring chromosomes harboring 8q24 amplicons. Immuno-FISH co-hybridization results in cases MLL_11929 (a), MLL_11933 (b), \#18 (c), and \#30 (d) display ring chromosomes with MYC amplifications (green) positive for CENP-A (red) but negative for alpha-satellite DNA (blue). Square boxes at the bottom left of each panel show the DAPI image of the rings

TCGA [0.7 and 0.2 for CS and FM, respectively; $U$-test $p$ $=0.004792(\mathrm{CS})$ and $p=1.116 \mathrm{e}-08(\mathrm{FM})]$ (Supplementary Figure S4c-d).

\section{AML-amp cases show a peculiar gene signature associated with $8 \mathrm{q} 24$ chimeras}

To investigate genes differentially expressed in AML-amp cases, we performed a DEA by comparing six AML-amp cases with six AML-NK samples from TCGA. Globally, we found 853 differentially expressed genes among which only two mapped in the 8q24 region, namely, MTSSI and RPI1532M24.1 (Supplementary Table S5A). Gene enrichment analysis showed that differentially expressed genes upregulated in AML-amp versus control cases displayed functions related to myeloid cell differentiation and homeostasis as well as chromatin assembly, nucleosome organization, histone methylation, and gene silencing (Supplementary Table S5B-C).

Unsupervised hierarchical clustering analysis of all the 8q24 genes in the 20 AML-amp cases revealed a high level of sample heterogeneity and clearly showed the presence of two major subgroups, denoted Group A (5 cases) and Group B (15 cases) (Fig. 4a). To ascertain the reason(s) for these two groups, we compared age, gender, cytogenetic features, and number of chimeras and genomic breakpoints involving the 8q24 region between the groups. This revealed a significant difference in the number of chimeras and genomic breakpoints (Fig. 4b). In addition, there was a positive correlation between the number of genomic breakpoints and the number of chimeras (identified by both CS and FM), indicating a close association between genomic complexity and transcriptomic plasticity of fusion transcripts (Fig. 4bc). The DEA of Group A versus Group B samples identified nine differentially expressed genes mapping in the 8q24 region, which were upregulated in Group A (Supplementary Table S5D). Among these, we found the IncRNA PVTI and the coding NSMCE2, which was the most significantly overexpressed gene in Group A $\left(\mathrm{FDR}=10^{-18}\right)$.

\section{Mutations in AML-amp cases affect genes involved in cell division and chromatin modifications}

We identified 467 SNVs/indels affecting 432 genes (Supplementary Table S6A), of which 19 were involved in two or more samples (Supplementary Table S6B), and 95 grouped into 31 gene families (Supplementary Table S6C). The most frequently mutated genes in the AML-amp cases were TET2 (50\% of the cases), DNMT3A (25\%), TP53 (15\%), and $K M T 2 C$ (alias $M L L 3,15 \%$ ). Pathway analysis grouped the 432 mutated genes into four main pathways: cell cycle, TP53 downstream pathway, chromatin modifications, and kinases (Supplementary Table S6D). These results indicate that most of the mutated genes are involved in cell division and epigenetic chromatin modifications.

\section{Overexpression of circular PVT1 is associated with high PVT1 CN amplification}

By qPCR, we demonstrated the strong upregulation of circPVT1, generated by the circularization of $P V T 1$ exon 2, in AML-amp cases with amplicons including PVT1 compared with AML-amp cases in which PVT1 was not amplified (as 07B60) or AML-NK samples (Fig. 5a). In AML cell lines, a slight upregulation of circPVT1 was observed only in the two cell lines carrying more than five copies of PVT1 (GF-D8 and KG-1). The PVT1 linear transcript also showed an upregulation in $P V T 1$-amplified versus not amplified AML cases, but this difference disappeared in AML cell lines where it was similarly expressed regardless of $P V T 1 \mathrm{CN}$ state (Fig. 5b).

\section{Discussion}

Our study focused on the comprehensive genomic and transcriptomic characterization of 8q24 amplifications in 


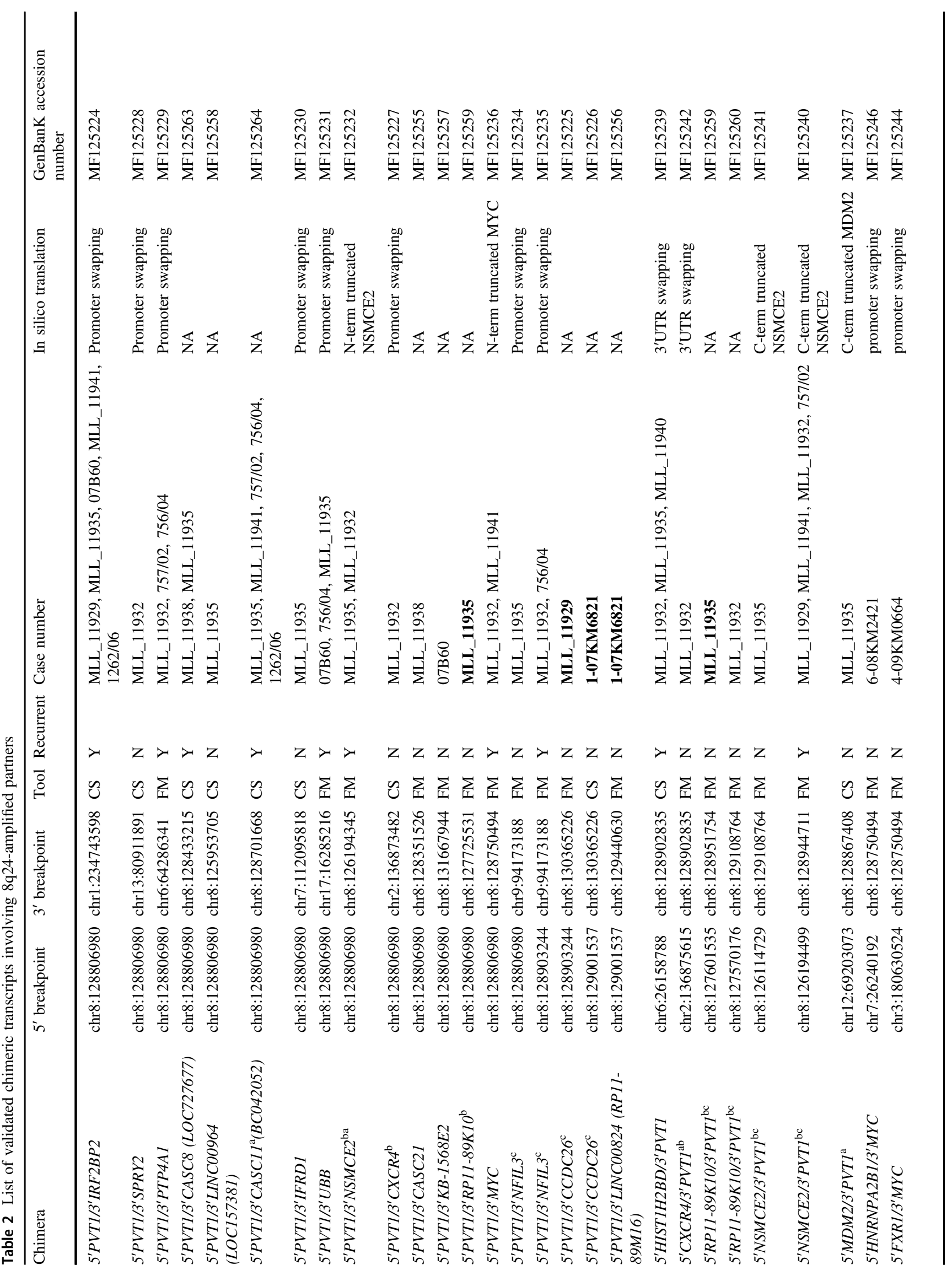


AML. Overall, $65 \%$ of cases showed evidence of amplicon heterogeneity. We previously observed the occurrence of distinct cell subpopulations harboring different $M Y C$ amplifications [9] and here confirmed at the singlenucleotide level.

Except for that of breakpoint clustering, we found that none of the valuable chromothripsis criteria is verified in our AML-amp cases. This is in accordance with our previous results on seven tumor cell lines with 8q24 amplicon heterogeneity, which excluded the chromothripsis model as involved in $\mathrm{dmin} / \mathrm{hsr}$ genesis [7], in contrast with previous literature $[5,6]$. As a matter of fact, breakpoint clustering might also result from events occurring at separate temporal stages of the amplicon evolution [18]. Additionally, in 48\% of cases we observed deletions corresponding to the amplified regions, not accompanied by any other rearrangement of the del(8) chromosome. Overall, these findings suggested a multi-step pathway in dmin/hsr genesis, indicating the episome model as the most reliable mechanism underlying 8q24 dmin genesis in AML, as we already proposed $[9,14]$.

8q24 amplifications also occurred as hsr and/or ring chromosomes in some of our cases, confirming that 8q24 amplicon architecture is shared among all these cytogenetic structures $[9,28]$. Interestingly, the discovery of neocentromeres on ring chromosomes is an unprecedented result, since ectopic centromeres emerging at chromosomes composed by genomic amplifications were observed only in well-differentiated liposarcomas [29] and lung cancer [30] thus far. Centric ring chromosomes might represent an evolutionary step of scattered acentric dmin as well as hsr towards mitotic stabilization, providing an adaptive advantage to leukemia cells harboring 8q24 amplifications. With the availability of vital frozen cells, further investigation into the genomic architectures of these neocentromeres will help to better understand the evolutionary path of these amplified sequences.

In this study, we focused on the identification of chimeric transcripts that originated from the 8q24-amplified genes. To date, $8 \mathrm{q} 24$ amplifications have already been associated with two fusion genes, namely PVTI-NSMCE2 and BF104016-NSMCE2, found respectively in an AML case and in the HL60 cell line [31]. Although the latter was not identified by neither WGS [7] nor RNA-seq in our research, we confirmed the occurrence of the $5^{\prime} P V T 1 / 3^{\prime} N S M C E 2$ fusion in our data set, along with its reciprocal $5^{\prime} N S M C E 2 /$ $3^{\prime} P V T 1$. The lncRNA PVT1 was described as a hotspot target of genomic breakpoints in 8q24 amplifications [7, 29, 32-34]. Notably, gain of $P V T 1$ expression is required for high MYC protein levels in 8q24-amplified human cancer cells [35]. Indeed, 26 out of 35 validated chimeras in our AML-amp cases involved this IncRNA, and most of them were recurrent in more cases and shared the same 

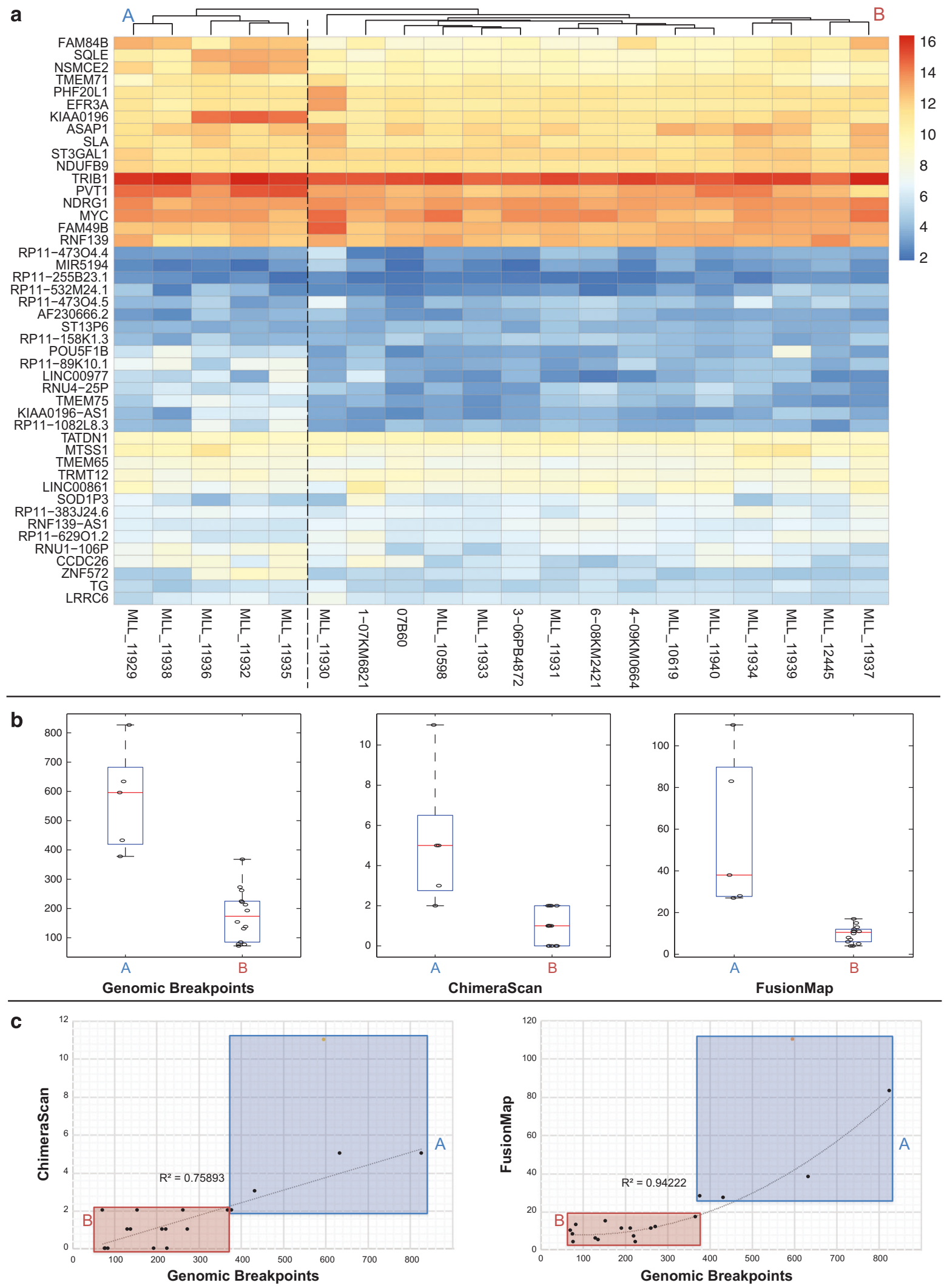
breakpoint position. Many of the 8q24 chimeras we identified lacked genomic support and were associated with $\mathrm{dmin} / \mathrm{hsr}$ amplification. Overall, the 8q24-amplified genes were shown to be prone to originate chimeric transcripts, often with not-amplified genes as partners. Our evidence indicates that they might be originated by alternative mechanisms to those based on genomic rearrangements. Post-transcriptional events, such as trans-splicing or cissplicing between adjacent genes (cis-SAGe) [36-40], might be an intriguing possibility that, however, needs further experimental support. The potential oncogenic role of these chimeras specifically accompanying 8q24 amplifications will require further investigation.

Notably, we found that AML-amp cases with the highest numbers of chimeras showed a peculiar 8q24 gene expression signature characterized by the overexpression of both PVT1 and NSMCE2, frequently found in our 8q24 chimeric transcripts. Unfortunately, such patient clustering cannot be directly correlated to disease prognostic indications, since most of Group A patients were lost during follow-up and lacked information about treatment and overall survival. However, we could envisage a possible prognostic implication given that Group A comprised three out the five cases with complex karyotype and TP53 mutation/17p13 loss, which are notoriously associated with a poorer outcome in AML [3]. Interestingly, NSMCE2, which was overexpressed in Group A cases, is required for DNA double-strand break repair and genomic integrity maintenance [41, 42]. Its depletion was shown to sensitize HeLa cells to DNA damage-induced apoptosis [43] suggesting that, alternatively, its overexpression could make tumor cells more resistant to the DNA damage induced by their wide genomic rearrangements.

Moreover, we characterized the gene signature and pathways specifically associated with the presence of $8 \mathrm{q} 24$ amplifications and found that the biological processes affected by these dysregulated genes are related to cell division and chromatin architecture. In particular, we observed the upregulation of genes involved in myeloid and erythroid cell development and differentiation, such as $C E B P A$ [44] and the key erythroid transcription factors GATA1 [45], TAL1, and KLF1 [46]. According to the literature [47], $K L F 1$ overexpression may be related to the simultaneous presence of TET2 and DNMT3A mutations in the two AML-amp cases investigated by our DEA. DNMT3A and TP53 frequencies are in line with those already reported in AML without 8q24 amplifications (30\% and $13 \%$, respectively) [48]. Conversely, TET2 and KMT2C mutations were more frequent in our cohort than in AML without amplifications (23\% and $0.5 \%$, respectively) [48, 49], although with no dysregulation at the expression level with respect to AML-NK samples. Interestingly, AML-amp cases also showed upregulated genes involved in chromatin assembly, nucleosome positioning, and H3K27 histone trimethylation, such as the replication-dependent histone genes HISTIHIB, HISTIHIC, HISTIHID, HISTIHIE, HIST1H2BG, HIST1H2BF, HIST1H3E (all mapping within the HIST1 cluster at 6p22-p21.3), and the replication independent HIFX (3q21.3). Considering that histone $\mathrm{H} 1$ and related linker histones are important for maintenance of higher-order chromatin structure and gene expression regulation [50], their significant upregulation in our cohort is of interest and might shed light on the chromatin compaction within dmin chromosomes.

Finally, the 8q24 commonly amplified genes, including $M Y C, P V T 1$, and TRIB1, showed a variegated expression pattern, consistent with previous reports [3, 9, 13, 14], thus confirming that they are not the real targets of this amplification type.

Remarkably, the transcriptional plasticity observed in our AML-amp cohort also includes circRNAs originated by back-splicing events [51], resistant to exonucleolytic degradation [52], and accumulating at relatively high levels within cells [47]. They have been proposed to act as microRNA sponges, platforms for protein interactions, transcriptional regulators of their linear counterparts, or, as recently demonstrated, as protein translators [53-57]. Concerning genes in 8q24 region, circRNAs from MTSS1, NSMCE2, FAM84B, POU5F1B, MYC, PVT1, and FAM49B have already been cataloged in the CircInteractome database (http://circinteractome.nia.nih.gov) [58]. circPVT1, generated from exon 2 of $P V T 1$, was found as highly expressed in HeLa cervical cancer cells, human proliferating fibroblasts [59], and gastric cancer cells often accompanied by 8q24 amplifications [53]. Notably, we found a strict correlation between $P V T 1 \mathrm{CN}$ gain and the upregulation of this circPVTl in both AML-amp cases and cell lines. The NB4 cell line was an exception, since it did not show overexpression of $\operatorname{circPVT1}$ despite its gene amplification; nonetheless, here, as well as in case 07B60, we cannot exclude the presence of additional $P V T 1$ alterations preventing the detection of the circRNA. $\operatorname{circPVT1}$ was proposed to act as a molecular sponge for tumor suppressor microRNAs, such as those belonging to let-7 [59] or miR125 families [53]. Further investigation into its role in AML-amp cases will therefore be required.

Fig. 4 Chimeric transcripts significantly associated with a peculiar gene expression profile at amplified regions and breakpoints. a Heatmap of 8 q24 gene expression levels in our AML-amp cohort. b Box and Whisker plots illustrating differences in the number of breakpoints and chimeras in 8q24 between Groups A and B. c Correlation analysis between the number of genomic breakpoints and chimeras in the 8q24 region identified by CS (left) and FM (right) 
Fig. 5 Circular and linear $P V T 1$ expression. qPCR results obtained for $\operatorname{circPVT1}$ (a) and linear PVT1 (b) in AML cases and cell lines. In red and green, respectively, samples with and without $P V T 1$ exon 2 gain. A pool of nine AML-NK cases was used as calibrator. NTC no template control

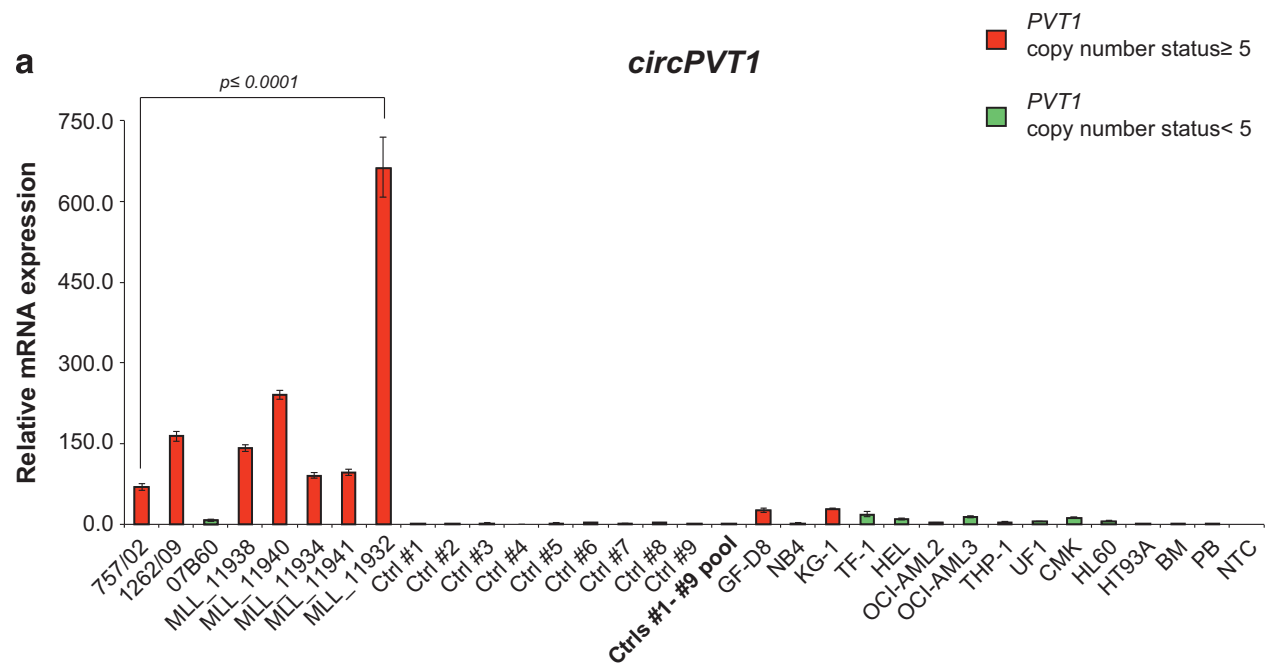

b

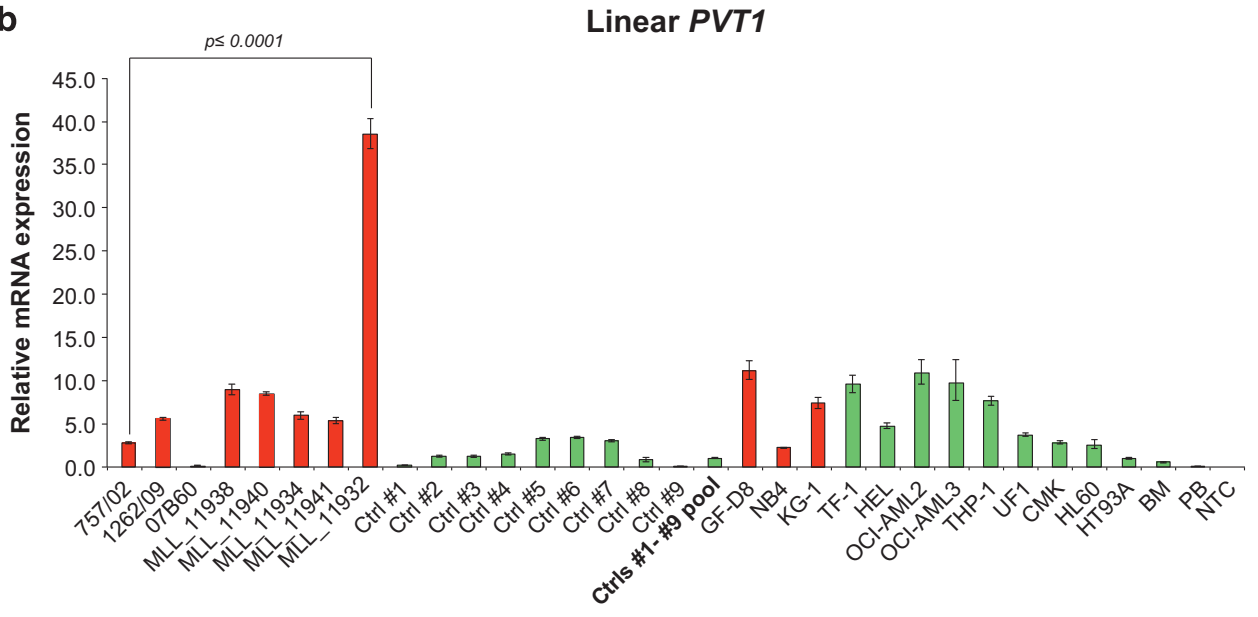

In summary, this work adds an important piece in the puzzle of the genomic and transcriptomic impact of 8q24 amplifications in AML. The amplicon molecular heterogeneity and the deletion of the corresponding amplified regions observed in the majority of cases ruled out chromothripsis as a potential mechanism behind their origin. Moreover, the neocentromeric seeding observed on ring chromosomes, harboring tandemly amplified sequences, would be engaged to stabilize collapsed acentric dmin, providing a selective advantage to leukemia cells. Finally, the finding of post-transcriptional chimeras and circRNAs involving 8q24-amplified genes in AML-amp cases could open new scenarios in the understanding of the biological mechanisms behind $M Y C$ amplifications in leukemia.

\section{Data availability}

SNP array data are available at EMBL-EBI ArrayExpress repository, under accession number E-MTAB-5372 (http://www.ebi.ac.uk/arrayexpress/experiments/E-MTAB5372/). WGS and RNA-seq data are available at NCBI
Short Read Archive (https://www.ncbi.nlm.nih.gov/sra) under accession number PRJNA386992. Validated SV sequences and RT-PCR validated fusion junction sequences were submitted to GenBank repository (http://www.ncbi. nlm.nih.gov/genbank/), under accession numbers MF084292-MF084375, and MF125224-MF125264 accession numbers, respectively.

Acknowledgements This work was supported by the AIRC (Associazione Italiana per la Ricerca sul Cancro; AIRC IG no. 14078 and 15413 to GM and CTS, respectively), the Italian Ministry of Health (Young researchers Call GR-2011-02352026 to IC), and the "5 $\times$ 1000 " voluntary contributions to GM. We thank Prof. Bertil Johansson and Prof. David Gisselsson for critical review of the manuscript.

Author contributions ALA and CTS conceived the study and wrote the manuscript, with assistance by DTo, IC, MS, GMe, and BJ. ALA, PDA, IC, MS, and GDB analyzed sequencing data and performed and reviewed bioinformatics and statistical analyses. AL, AT, BA, MP, $\mathrm{GMe}$, and DTr designed and performed PCR experiments. AT, DTr, MP, and DTo performed Sanger sequencing and FISH validation of data. OP and MC performed and analyzed SNP array data. DTo and DTr performed immuno-FISH experiments. AD and AT performed mutational analysis under the supervision of $\mathrm{LB}$. GD and $\mathrm{AL}$ 
performed circRNA RT-qPCR and pull-down assays. GMa, CH, LB, DM, JS, and NVR provided biological samples and clinical data. All authors read and approved the final manuscript.

\section{Compliance with ethical standards}

Conflict of interest The authors declare that they have no conflict of interest.

Open Access This article is licensed under a Creative Commons Attribution-NonCommercial-NoDerivatives 4.0 International License, which permits any non-commercial use, sharing, distribution and reproduction in any medium or format, as long as you give appropriate credit to the original author(s) and the source, and provide a link to the Creative Commons license. You do not have permission under this license to share adapted material derived from this article or parts of it. The images or other third party material in this article are included in the article's Creative Commons license, unless indicated otherwise in a credit line to the material. If material is not included in the article's Creative Commons license and your intended use is not permitted by statutory regulation or exceeds the permitted use, you will need to obtain permission directly from the copyright holder. To view a copy of this license, visit http://creativecommons.org/licenses/by-nc-nd/4.0/.

\section{References}

1. Turner KM, Deshpande V, Beyter D, Koga T, Rusert J, Lee C, et al. Extrachromosomal oncogene amplification drives tumour evolution and genetic heterogeneity. Nature. 2017;543:122-5.

2. Mitelman F, Johansson B, Mertens FE Mitelman Database of Chromosome Aberrations and Gene Fusions in Cancer. 2017.

3. Huh YO, Tang G, Talwalkar SS, Khoury JD, Ohanian M, BuesoRamos CE, et al. Double minute chromosomes in acute myeloid leukemia, myelodysplastic syndromes, and chronic myelomonocytic leukemia are associated with micronuclei, MYC or MLL amplification, and complex karyotype. Cancer Genet. 2016;209:313-20.

4. Sait SN, Qadir MU, Conroy JM, Matsui S, Nowak NJ, Baer MR. Double minute chromosomes in acute myeloid leukemia and myelodysplastic syndrome: identification of new amplification regions by fluorescence in situ hybridization and spectral karyotyping. Genes Chromosomes Cancer. 2002;34:42-47.

5. Stephens PJ, Greenman CD, Fu B, Yang F, Bignell GR, Mudie $\mathrm{LJ}$, et al. Massive genomic rearrangement acquired in a single catastrophic event during cancer development. Cell. 2011;144:27-40.

6. Rausch T, Jones DT, Zapatka M, Stutz AM, Zichner T, Weischenfeldt $\mathrm{J}$, et al. Genome sequencing of pediatric medulloblastoma links catastrophic DNA rearrangements with TP53 mutations. Cell. 2012;148:59-71.

7. L'Abbate A, Macchia G, D'Addabbo P, Lonoce A, Tolomeo D, Trombetta D, et al. Genomic organization and evolution of double minutes/homogeneously staining regions with MYC amplification in human cancer. Nucleic Acids Res. 2014;42:9131-45.

8. Schwab M. Oncogene amplification in solid tumors. Semin Cancer Biol. 1999;9:319-25.

9. Storlazzi CT, Fioretos T, Surace C, Lonoce A, Mastrorilli A, Strombeck B, et al. MYC-containing double minutes in hematologic malignancies: evidence in favor of the episome model and exclusion of MYC as the target gene. Hum Mol Genet. 2006;15:933-42.

10. Storlazzi CT, Lonoce A, Guastadisegni MC, Trombetta D, D'Addabbo P, Daniele G, et al. Gene amplification as double minutes or homogeneously staining regions in solid tumors: origin and structure. Genome Res. 2010;20:1198-206.

11. Santarius T, Shipley J, Brewer D, Stratton MR, Cooper CS. A census of amplified and overexpressed human cancer genes. Nat Rev Cancer. 2010;10:59-64.

12. Paulsson K, Lassen C, Kuric N, Billstrom R, Fioretos T, Tanke $\mathrm{HJ}$, et al. MYC is not overexpressed in a case of chronic myelomonocytic leukemia with MYC-containing double minutes. Leukemia. 2003;17:813-5.

13. Storlazzi CT, Fioretos T, Paulsson K, Strombeck B, Lassen C, Ahlgren $\mathrm{T}$, et al. Identification of a commonly amplified $4.3 \mathrm{Mb}$ region with overexpression of $\mathrm{C} 8 \mathrm{FW}$, but not MYC in MYCcontaining double minutes in myeloid malignancies. Hum Mol Genet. 2004;13:1479-85.

14. Micale L, Augello B, Daniele G, Macchia G, L'Abbate A, Muehlematter D, et al. Amplification of the $G$ allele at SNP rs6983267 in 8q24 amplicons in myeloid malignancies as cause of the lack of MYC overexpression? Blood Cells Mol Dis. 2011;47:259-61.

15. Trazzi S, Perini G, Bernardoni R, Zoli M, Reese JC, Musacchio A, et al. The C-terminal domain of CENP-C displays multiple and critical functions for mammalian centromere formation. PLoS ONE. 2009;4:e5832.

16. Macchia G, Nord KH, Zoli M, Purgato S, D'Addabbo P, Whelan $\mathrm{CW}$, et al. Ring chromosomes, breakpoint clusters, and neocentromeres in sarcomas. Genes Chromosomes Cancer. 2015;54:156-67.

17. Rausch T, Zichner T, Schlattl A, Stutz AM, Benes V, Korbel JO. DELLY: structural variant discovery by integrated paired-end and split-read analysis. Bioinformatics. 2012;28:i333-i339.

18. Korbel JO, Campbell PJ. Criteria for inference of chromothripsis in cancer genomes. Cell. 2013;152:1226-36.

19. Iyer MK, Chinnaiyan AM, Maher CA. ChimeraScan: a tool for identifying chimeric transcription in sequencing data. Bioinformatics. 2011;27:2903-4.

20. Ge H, Liu K, Juan T, Fang F, Newman M, Hoeck W. FusionMap: detecting fusion genes from next-generation sequencing data at base-pair resolution. Bioinformatics. 2011;27:1922-8.

21. McKenna A, Hanna M, Banks E, Sivachenko A, Cibulskis K, Kernytsky A, et al. The Genome Analysis Toolkit: a MapReduce framework for analyzing next-generation DNA sequencing data. Genome Res. 2010;20:1297-303.

22. Koboldt DC, Chen K, Wylie T, Larson DE, McLellan MD, Mardis ER, et al. VarScan: variant detection in massively parallel sequencing of individual and pooled samples. Bioinformatics. 2009;25:2283-5.

23. Liberzon A, Subramanian A, Pinchback R, Thorvaldsdottir H, Tamayo P, Mesirov JP. Molecular signatures database (MSigDB) 3.0. Bioinformatics. 2011;27:1739-40.

24. Subramanian A, Tamayo P, Mootha VK, Mukherjee S, Ebert BL, Gillette MA, et al. Gene set enrichment analysis: a knowledgebased approach for interpreting genome-wide expression profiles. Proc Natl Acad Sci USA. 2005;102:15545-50.

25. Huang da W, Sherman BT, Lempicki RA. Systematic and integrative analysis of large gene lists using DAVID bioinformatics resources. Nat Protoc. 2009;4:44-57.

26. Chen J, Li Y, Zheng Q, Bao C, He J, Chen B, et al. Circular RNA profile identifies circPVT1 as a proliferative factor and prognostic marker in gastric cancer. Cancer Lett. 2016;388:208-19.

27. Pfaffl MW, Horgan GW, Dempfle L. Relative expression software tool (REST) for group-wise comparison and statistical analysis of relative expression results in real-time PCR. Nucleic Acids Res. 2002;30:e36.

28. Reddy KS. Double minutes (dmin) and homogeneously staining regions (hsr) in myeloid disorders: a new case suggesting that dmin form hsr in vivo. Cytogenet Genome Res. 2007;119:53-59. 
29. Garsed DW, Marshall OJ, Corbin VD, Hsu A, Di Stefano L, Schroder J, et al. The architecture and evolution of cancer neochromosomes. Cancer Cell. 2014;26:653-67.

30. Italiano A, Attias R, Aurias A, Perot G, Burel-Vandenbos F, Otto $\mathrm{J}$, et al. Molecular cytogenetic characterization of a metastatic lung sarcomatoid carcinoma: 9p23 neocentromere and 9p23-p24 amplification including JAK2 and JMJD2C. Cancer Genet Cytogenet. 2006;167:122-30.

31. Chinen Y, Sakamoto N, Nagoshi H, Taki T, Maegawa S, Tatekawa $\mathrm{S}$, et al. 8q24 amplified segments involve novel fusion genes between NSMCE2 and long noncoding RNAs in acute myelogenous leukemia. J Hematol Oncol. 2014;7:68.

32. Iwakawa R, Takenaka M, Kohno T, Shimada Y, Totoki Y, Shibata $\mathrm{T}$, et al. Genome-wide identification of genes with amplification and/or fusion in small cell lung cancer. Genes Chromosomes Cancer. 2013;52:802-16.

33. Nagoshi H, Taki T, Hanamura I, Nitta M, Otsuki T, Nishida K, et al. Frequent PVT1 rearrangement and novel chimeric genes PVT1-NBEA and PVT1-WWOX occur in multiple myeloma with 8q24 abnormality. Cancer Res. 2012;72:4954-62.

34. Northcott PA, Shih DJ, Peacock J, Garzia L, Morrissy AS, Zichner $\mathrm{T}$, et al. Subgroup-specific structural variation across 1000 medulloblastoma genomes. Nature. 2012;488:49-56.

35. Tseng YY, Moriarity BS, Gong W, Akiyama R, Tiwari A, Kawakami H, et al. PVT1 dependence in cancer with MYC copynumber increase. Nature. 2014;512:82-86.

36. Babiceanu M, Qin F, Xie Z, Jia Y, Lopez K, Janus N, et al. Recurrent chimeric fusion RNAs in non-cancer tissues and cells. Nucleic Acids Res. 2016;44:2859-72.

37. Chen J, Bardes EE, Aronow BJ, Jegga AG. ToppGene suite for gene list enrichment analysis and candidate gene prioritization. Nucleic Acids Res. 2009;37:W305-311.

38. Qin F, Song Z, Babiceanu M, Song Y, Facemire L, Singh R, et al. Discovery of CTCF-sensitive Cis-spliced fusion RNAs between adjacent genes in human prostate cells. PLoS Genet. 2015;11: e1005001.

39. Zaphiropoulos PG. Trans-splicing in higher eukaryotes: implications for cancer development? Front Genet. 2011;2:92.

40. Zhang Y, Gong M, Yuan H, Park HG, Frierson HF, Li H. Chimeric transcript generated by cis-splicing of adjacent genes regulates prostate cancer cell proliferation. Cancer Discov. 2012;2:598-607.

41. Stephan AK, Kliszczak M, Morrison CG. The Nse2/Mms 21 SUMO ligase of the Smc5/6 complex in the maintenance of genome stability. FEBS Lett. 2011;585:2907-13.

42. Verver DE, Zheng Y, Speijer D, Hoebe R, Dekker HL, Repping S. et al. Non-SMC element 2 (NSMCE2) of the SMC5/6 complex helps to resolve topological stress. Int J Mol Sci. 2016;17:1782

43. Potts PR, Yu H. Human MMS21/NSE2 is a SUMO ligase required for DNA repair. Mol Cell Biol. 2005;25:7021-32.
44. Ohlsson E, Schuster MB, Hasemann M, Porse BT. The multifaceted functions of C/EBPalpha in normal and malignant haematopoiesis. Leukemia. 2016;30:767-75.

45. Shimizu R, Engel JD, Yamamoto M. GATA1-related leukaemias. Nat Rev Cancer. 2008;8:279-87.

46. Tallack MR, Magor GW, Dartigues B, Sun L, Huang S, Fittock $\mathrm{JM}$, et al. Novel roles for KLF1 in erythropoiesis revealed by mRNA-seq. Genome Res. 2012;22:2385-98.

47. Zhang Y, Xue W, Li X, Zhang J, Chen S, Zhang JL, et al. The biogenesis of nascent circular RNAs. Cell Rep. 2016;15:611-24.

48. Rose D, Haferlach T, Schnittger S, Perglerova K, Kern W, Haferlach C. Subtype-specific patterns of molecular mutations in acute myeloid leukemia. Leukemia. 2017;31:11-17.

49. Garg M, Nagata Y, Kanojia D, Mayakonda A, Yoshida K, Haridas Keloth S, et al. Profiling of somatic mutations in acute myeloid leukemia with FLT3-ITD at diagnosis and relapse. Blood. 2015;126:2491-501.

50. Harshman SW, Young NL, Parthun MR, Freitas MA. H1 histones: current perspectives and challenges. Nucleic Acids Res. 2013;41:9593-609.

51. Sibley CR, Blazquez L, Ule J. Lessons from non-canonical splicing. Nat Rev Genet. 2016;17:407-21.

52. Enuka Y, Lauriola M, Feldman ME, Sas-Chen A, Ulitsky I, Yarden Y. Circular RNAs are long-lived and display only minimal early alterations in response to a growth factor. Nucleic Acids Res. 2016;44:1370-83.

53. Chen LL. The biogenesis and emerging roles of circular RNAs. Nat Rev Mol Cell Biol. 2016;17:205-11.

54. Jeck WR, Sharpless NE. Detecting and characterizing circular RNAs. Nat Biotechnol. 2014;32:453-61.

55. Legnini I, Di Timoteo G, Rossi F, Morlando M, Briganti F, Sthandier O, et al. Circ-ZNF609 is a circular RNA that can be translated and functions in myogenesis. Mol Cell. 2017;66:22-37. e29

56. Salzman J. Circular RNA expression: its potential regulation and function. Trends Genet. 2016;32:309-16.

57. Yang Y, Fan X, Mao M, Song X, Wu P, Zhang Y, et al. Extensive translation of circular RNAs driven by N6-methyladenosine. Cell Res. 2017;27:626-41.

58. Dudekula DB, Panda AC, Grammatikakis I, De S, Abdelmohsen K, Gorospe M. CircInteractome: A web tool for exploring circular RNAs and their interacting proteins and microRNAs. RNA Biol. 2016;13:34-42.

59. Panda AC, Grammatikakis I, Kim KM, De S, Martindale JL, Munk R, et al. Identification of senescence-associated circular RNAs (SAC-RNAs) reveals senescence suppressor CircPVT1. Nucleic Acids Res. 2016;45:4021-35. 